\title{
The universality of homogeneous polynomial forms and critical limits
}

\author{
Shuyang Bai Murad S. Taqqu
}

June 12, 2018

\begin{abstract}
Nourdin et al. [9] established the following universality result: if a sequence of off-diagonal homogeneous polynomial forms in i.i.d. standard normal random variables converges in distribution to a normal, then the convergence also holds if one replaces these i.i.d. standard normal random variables in the polynomial forms by any independent standardized random variables with uniformly bounded third absolute moment. The result, which was stated for polynomial forms with a finite number of terms, can be extended to allow an infinite number of terms in the polynomial forms. Based on a contraction criterion derived from this extended universality result, we prove a central limit theorem for a strongly dependent nonlinear processes, whose memory parameter lies at the boundary between short and long memory.
\end{abstract}

\section{Introduction}

In Nourdin et al. 9], a universality result was established for the following off-diagonal homogeneous polynomial form

$$
Q_{k}\left(N_{n}, f_{n}, \mathbf{X}\right):=\sum_{1 \leq i_{1}, \ldots, i_{k} \leq N_{n}} f_{n}\left(i_{1}, \ldots, i_{k}\right) X_{i_{1}} \ldots X_{i_{k}},
$$

where $f_{n}$ is a sequence of symmetric functions on $\mathbb{Z}_{+}^{k}$ vanishing on the diagonals $\left(f_{n}\left(i_{1}, \ldots, i_{k}\right)=0\right.$ if $i_{p}=i_{q}$ for some $p \neq q)$, and $\mathbf{X}=\left(X_{1}, X_{2}, \ldots\right)$ is a sequence of standardized independent random variables, and $N_{n}$ is a finite sequence such that $N_{n} \rightarrow \infty$ as $n \rightarrow \infty$.

The universality result says that if $\mathbf{Z}=\left(Z_{1}, Z_{2}, \ldots\right)$ is an i.i.d. standard normal sequence and $Q_{k}\left(N_{n}, f_{n}, \mathbf{Z}\right)$ converges weakly to a normal distribution as $n \rightarrow \infty$, then the same weak convergence to normal holds if $\mathbf{Z}$ is replaced by $\mathbf{X}$, where $\mathbf{X}$ is any standardized independent sequence with some uniform higher moment bound.

It is natural to try to eliminate the finiteness of $N_{n}$ in the preceding result. This extension was mentioned in Remark 1.13 of Nourdin et al. [9], but was not explicitly done. One would encounter a number of difficulties if one were to extend the method of proof used for finite $N_{n}$ to $N_{n}=\infty$. We will note, however, that this extension can be easily achieved using a simple approximation argument. We find it valuable to have such an extension and the corresponding contraction criterion (Theorem 2.6) since it can be directly applied to limit theorems in the context of long memory.

We consider such an application in Section 3 where we suppose that

$$
f_{N}\left(i_{1}, \ldots, i_{k}\right)=\frac{1}{A(N)} \sum_{n=1}^{N} a\left(n-i_{1}, \ldots, n-i_{k}\right) 1_{\left\{-\infty<i_{1}<n, \ldots,-\infty<i_{k}<n\right\}},
$$

Key words Universality, Wiener chaos, long memory, long-range dependence

2010 AMS Classification: 60F05 
and where the function $a(\cdot)$ behaves essentially like a homogeneous function with exponent $\alpha$. The resulting polynomial form $Q_{k}\left(f_{N}\right)$ is then the partial sum of a stationary process. The exponent $\alpha$ is chosen in such a way that the corresponding stationary process lives on the boundary between short and long memory. We use the contraction criterion to prove that a central limit theorem holds but with the nonstandard normalization $\sqrt{N \ln N}$. This delicate case seems difficult to treat otherwise.

The paper is organized as follows. In Section 2, we state the and prove the extension of the universality result (Theorem 2.1), and as a byproduct, a criterion for asymptotic normality (Theorem 2.6). In Section 3.1. we state the critical limit theorem obtained by applying the criterion. In Section 3.3 and 3.4 we give the proofs.

\section{Universality of homogeneous polynomial forms}

Let $\ell^{2}\left(\mathbb{Z}^{k}\right), k \geq 1$, denote the space of symmetric square summable functions on $\mathbb{Z}^{k}$ vanishing on the diagonals equipped with the discrete $L^{2}$ norm. Let $\mathbf{X}=\left(X_{1}, X_{2}, \ldots\right)$ be a sequence of independent random variables satisfying $\mathbb{E} X_{i}=0$ and $\mathbb{E} X_{i}^{2}=1$. By modifying the notation (1), one defines for $f \in \ell^{2}\left(\mathbb{Z}^{k}\right)$ :

$$
Q_{k}(f, \mathbf{X}):=\sum_{-\infty<i_{1}, \ldots, i_{k}<\infty} f\left(i_{1}, \ldots, i_{k}\right) X_{i_{1}} \ldots X_{i_{k}}
$$

One has

$$
\mathbb{E} Q_{k}(f, \mathbf{X})=0 .
$$

Consider now two homogeneous polynomial forms $Q_{k_{1}}\left(f_{1}, \mathbf{X}\right)$ and $Q_{k_{2}}\left(f_{2}, \mathbf{X}\right)$, where $f_{1} \in \ell^{2}\left(\mathbb{Z}^{k_{1}}\right)$ and $f_{2} \in \ell^{2}\left(\mathbb{Z}^{k_{2}}\right)$. Then the covariance of $Q_{k_{1}}\left(f_{1}, \mathbf{X}\right)$ and $Q_{k_{2}}\left(f_{2}, \mathbf{X}\right)$ is

$$
\begin{aligned}
\left\langle f_{1}, f_{2}\right\rangle & :=\mathbb{E} Q_{k}\left(f_{1}, \mathbf{X}\right) Q_{k}\left(f_{2}, \mathbf{X}\right) \\
& = \begin{cases}k ! \sum_{-\infty<i_{1}, \ldots, i_{k}<\infty} f_{1}\left(i_{1}, \ldots, i_{k}\right) f_{2}\left(i_{1}, \ldots, i_{k}\right), & \text { if } k_{1}=k_{2}=k ; \\
0 & \text { if } k_{1} \neq k_{2} .\end{cases}
\end{aligned}
$$

We then have the following extension of Nourdin et al. [9] Theorem 1.2:

Theorem 2.1. For each $j=1, \ldots, m$, suppose that $k_{j} \geq 2$, and let $f_{n, j}(\cdot)$ be a sequence of functions in $\ell^{2}\left(\mathbb{Z}^{k_{j}}\right)$. Let $\Sigma$ be an $m \times m$ symmetric non-negative definite matrix whose each diagonal entry is positive. Assume in addition that

$$
\sup _{n} \sum_{-\infty<i_{1}, \ldots, i_{k_{j}}<\infty} f_{n, j}\left(i_{1}, \ldots, i_{k_{j}}\right)^{2}<\infty .
$$

Then the following two statements are equivalent:

1. For every sequence $\mathbf{X}=\left(X_{1}, X_{2}, \ldots\right)$ where $X_{1}, X_{2}, \ldots$ are independent random variables satisfying $\mathbb{E} X_{i}=0, \mathbb{E} X_{i}^{2}=1$, and

$$
\sup _{i} \mathbb{E}\left|X_{i}\right|^{3}<\infty,
$$

the following joint weak convergence to a multivariate normal distribution holds:

$$
\left(Q_{k_{j}}\left(f_{n, j}, \mathbf{X}\right)\right)_{j=1}^{m} \stackrel{d}{\rightarrow} N(\mathbf{0}, \Sigma)
$$

2. For a sequence $\mathbf{Z}=\left(Z_{1}, Z_{2}, \ldots\right)$ of i.i.d. standard normal random variables, the following joint weak convergence to a multivariate normal distribution holds:

$$
\left(Q_{k_{j}}\left(f_{n, j}, \mathbf{Z}\right)\right)_{j=1}^{m} \stackrel{d}{\rightarrow} N(\mathbf{0}, \Sigma) .
$$


Remark 2.2. Condition (4) can be re-expressed as

$$
\sup _{n} \mathbb{E} Q_{k_{j}}\left(f_{n, j}, \mathbf{Z}\right)^{2}=k_{j} ! \sup _{n} \sum_{-\infty<i_{1}, \ldots, i_{k_{j}}<\infty} f_{n, j}\left(i_{1}, \ldots, i_{k_{j}}\right)^{2}<\infty .
$$

Remark 2.3. One can recover Nourdin et al. [9] Theorem 1.2 from Theorem[2.1 by replacing $f_{n, j}\left(i_{1}, \ldots, i_{k_{j}}\right)$ with $f_{n, j}\left(i_{1}, \ldots, i_{k_{j}}\right) 1_{1 \leq i_{1}, \ldots, i_{k_{j}} \leq N_{n}}\left(i_{1}, \ldots, i_{k_{j}}\right)$.

Remark 2.4. In the one dimensional case: $m=1$, one can relax the assumption (5) by $\sup _{i} \mathbb{E}\left|X_{i}\right|^{2+\delta}<\infty$ for any $\delta>0$. See Theorem 1.10 of Nourdin et al. [9].

Proof of Theorem [2.1] We need to prove that (77) implies (6). Define the $N_{n}$-truncated functions

$$
\tilde{f}_{n, j}\left(i_{1}, \ldots, i_{k_{j}}\right)=f_{n, j}\left(i_{1}, \ldots, i_{k_{j}}\right) 1_{\left\{-N_{n} \leq i_{1} \leq N_{n}, \ldots,-N_{n} \leq i_{k_{j}} \leq N_{n}\right\}}, \quad j=1, \ldots, m .
$$

For any $n \in \mathbb{Z}_{+}$, we can find $N_{n}$ large enough, so that for all $j=1, \ldots, m$,

$$
\mathbb{E}\left|Q_{k_{j}}\left(f_{n, j}, \mathbf{Z}\right)-Q_{k_{j}}\left(\tilde{f}_{n, j}, \mathbf{Z}\right)\right|^{2}=\mathbb{E}\left|Q_{k_{j}}\left(f_{n, j}, \mathbf{X}\right)-Q_{k_{j}}\left(\tilde{f}_{n, j}, \mathbf{X}\right)\right|^{2}=k_{j} !\left\|\tilde{f}_{n, j}-f_{n, j}\right\|_{\ell^{2}\left(\mathbb{Z}^{k_{j}}\right)}^{2} \leq \frac{1}{n} .
$$

Assume without loss of generality that $N_{n} \rightarrow \infty$ as $n \rightarrow \infty$. By (77) and (9), one has

$$
\left(Q_{k_{j}}\left(\tilde{f}_{n, j}, \mathbf{Z}\right)\right)_{j=1}^{m} \stackrel{d}{\rightarrow} N(\mathbf{0}, \Sigma)
$$

Using the original version of the universality result in Nourdin et al. 9] Theorem 1.2, one gets

$$
\left(Q_{k_{j}}\left(\tilde{f}_{n, j}, \mathbf{X}\right)\right)_{j=1}^{m} \stackrel{d}{\rightarrow} N(\mathbf{0}, \Sigma)
$$

The conclusion (6) follows from (9) and (10).

Remark 2.5. Using the same argument as in the preceding proof, one can eliminate the finiteness of $N_{n}$ in (11) in the following related universality results for homogeneous polynomial forms: (a) Theorem 1.12 of Nourdin et al. [9] concerning for convergence to a $\chi^{2}$ distribution; (b) Theorem 3.4 of Peccati and Zheng [11] which is the counterpart of Theorem 2.1 here with $Z_{i}$ 's being standardized Poisson random variables.

Theorem 2.1 gives rise to a practical criterion for the convergence (6). We first introduce the discrete contraction operator: for $f \in \ell^{p}\left(\mathbb{Z}^{p}\right)$ and $g \in \ell^{q}\left(\mathbb{Z}^{q}\right), p, q \geq 2$, we define

$$
\left(f \star_{r} g\right)\left(i_{1}, \ldots, i_{p+q-2 r}\right)=\sum_{j_{1}, \ldots, j_{r}=-\infty}^{\infty} f\left(j_{1}, \ldots, j_{r}, i_{1}, \ldots, i_{p-r}\right) g\left(j_{1}, \ldots, j_{r}, i_{p-r+1}, \ldots, i_{p+q-2 r}\right)
$$

for $r=0, \ldots, p \wedge q$, where in the case $r=0$ it is understood as the tensor product.

Theorem 2.6. Let $\left\{f_{n, j}(\cdot), n \in \mathbb{Z}_{+}\right\}$be a sequence of functions in $\ell^{2}\left(\mathbb{Z}^{k_{j}}\right)$ satisfying (4), $j=1, \ldots, m$, where $k_{j} \geq 2$. Let $\Sigma$ be an $m \times m$ symmetric non-negative definite matrix whose each diagonal entry is positive, such that

$$
\Sigma(i, j)=\lim _{n \rightarrow \infty}\left\langle f_{n, i}, f_{n, j}\right\rangle,
$$

where $\langle\cdot, \cdot\rangle$ is defined in (3). Then the following are equivalent:

1. For every $\mathbf{X}=\left(X_{1}, X_{2}, \ldots\right)$ with $X_{i}$ 's being independent random variables satisfying $\mathbb{E} X_{i}=0, \mathbb{E} X_{i}^{2}=1$ and $\sup _{i} \mathbb{E}\left|X_{i}\right|^{3}<\infty$, we have the following joint weak convergence to normal:

$$
\left(Q_{k_{j}}\left(f_{n, j}, \mathbf{X}\right)\right)_{j=1}^{m} \stackrel{d}{\rightarrow} N(\mathbf{0}, \Sigma) .
$$




\section{The following contractions are vanishing:}

$$
\lim _{n \rightarrow \infty}\left\|f_{n, j} \star_{r} f_{n, j}\right\|_{2 k_{j}-2 r}=0, \quad \text { for all } r=1, \ldots, k_{j}-1 \text { and all } j=1, \ldots, m \text {. }
$$

where $\|\cdot\|_{k}$ denotes the discrete $L^{2}$ norm on $\ell^{2}\left(\mathbb{Z}^{k}\right)$.

Proof. By Theorem 2.1, the statement 1 is equivalent to $\left(Q_{k_{j}}\left(f_{n, j}, \mathbf{Z}\right)\right)_{j=1}^{m} \stackrel{d}{\rightarrow} N(\mathbf{0}, \Sigma)$, where $\mathbf{Z}$ is a sequence of i.i.d. standard Gaussian variables. Note also that each $Q_{k_{j}}\left(f_{n, j}, \mathbf{Z}\right)$ can be expressed as a $k_{j}$-tuple WienerItô integral with respect to Brownian motion. For Wiener-Itô integrals, joint convergence to the normal is equivalent to marginal convergence, and marginal convergence is equivalent to the contraction relations. More precisely, by applying Theorem 6.2.3 and 5.2.7 of Nourdin and Peccati [8], one gets the equivalence to (14). See also Theorem 7.5 of Nourdin et al. [9].

Remark 2.7. We shall use the implication "Statement $2 \Rightarrow$ Statement 1" of the preceding theorem in the sequel. As for the reversed implication, namely, "Statement $1 \Rightarrow$ Statement 2", the stipulation "For every" is important here, as well as in Theorem 2.1, because there are random variables $X_{i}$ 's, for example Rademacher, that is $X_{i}= \pm 1$ with probability $1 / 2$ each, for which one may have convergence in (13) even when (14) does not hold (see Nourdin et al. [9], Section 1.6, p.1956).

Remark 2.8. One may wonder if the universality result extends to a continuous setting, namely, when $Q_{k}\left(f_{n}\right)$ is replaced by a multiple integral on a Borel measure space $(A, \mathcal{A}, \mu)$ :

$$
I_{k}\left(f_{n}, \xi\right)=\int_{A^{k}}^{\prime} f_{n}\left(x_{1}, \ldots, x_{k}\right) \xi\left(d x_{1}\right) \ldots \xi\left(d x_{k}\right),
$$

where $f \in L^{2}\left(A^{k}\right)$, the prime' indicates the exclusion of diagonals $x_{p}=x_{q}, p \neq q$, and $\xi(\cdot)$ is an independently scattered random measure with an atomless control measure $\mu(\cdot)$. Does $I_{k}\left(f_{n}, \xi\right)$ exhibits a similar universality phenomenon? Namely, if $I_{k}\left(f_{n}, \xi\right)$ converges in distribution to normal for a Gaussian $\xi(\cdot)$, does the convergence also hold for general class of $\xi(\cdot)$ with the same control measure $\mu(\cdot)$ ? It is known that the law of $\xi(\cdot)$ has to be infinitely divisible and $\xi(\cdot)$ admits the decomposition:

$$
\xi(B)=G(B)+\int_{\mathbb{R}} \int_{A} u 1_{B}(x) \widehat{N}(d u, d x),
$$

where $G(\cdot)$ is a Gaussian random measure on $A$ and $\widehat{N}(\cdot)$ is an independent compensated Poisson random measure on $\mathbb{R} \times A$. See Section 5.3 of Peccati and Taqqu [10] for more details.

One may think of adapting the approximation argument used in the proof of Theorem 2.1 to the multiple integral case, which would involve partitioning the space $A$ into subsets of small measure. The problem is that unlike the Gaussian part, the Poisson part does not scale as $\mu(B) \rightarrow 0$. To see this in the simplest situation, take $\xi(B)=\widehat{P}(B)$, where $\widehat{P}(\cdot)$ is a compensated Poisson random measure on $A$ with control measure $\mu(\cdot)$. Note that $\widehat{P}(B)+\mu(B)$ follows a Poisson distribution with mean $\mu(B)$. Since its cumulants are all equal to $\mu(B)$ (see (3.1.5) of Peccati and Taqqu [10]), and since the third moment of a centered random variable is equal to the third cumulant, one has $\mathbb{E}(\widehat{P}(B))^{3}=\mu(B)$. This means that although we have the standardization

$$
\mathbb{E}|\widehat{P}(B) / \sqrt{\mu(B)}|^{2}=1,
$$

we also have

$\lim _{\mu(B) \rightarrow 0} \mathbb{E}|\widehat{P}(B) / \sqrt{\mu(B)}|^{3}=\lim _{\mu(B) \rightarrow 0} \mathbb{E}|\widehat{P}(B)|^{3} \mu(B)^{-3 / 2} \geq \lim _{\mu(B) \rightarrow 0} \mathbb{E} \widehat{P}(B)^{3} \mu(B)^{-3 / 2}=\lim _{\mu(B) \rightarrow 0} \mu(B)^{-1 / 2}=\infty$.

This will violate condition (5) as the partition of $A$ becomes finer. In fact, one can show that $\widehat{P}(B) / \sqrt{\mu(B)} \rightarrow$ 0 in probability as $\mu(B) \rightarrow 0$, which means, in view of (16), that the uniform integrability of $|\widehat{P}(B) / \sqrt{\mu(B)}|^{2}$ fails. For further insights, see Rotar [12]. 


\section{Application: boundary between short and long memory}

\subsection{The setting}

Bai and Taqqu [1] considered the following discrete chaos processes:

$$
X(n)=\sum_{-\infty<i_{1}, \ldots, i_{k}<n} a\left(n-i_{1}, \ldots, n-i_{k}\right) \epsilon_{i_{1}} \ldots \epsilon_{i_{k}},
$$

where $k \geq 2, a(\cdot): \mathbb{Z}_{+}^{k} \rightarrow \mathbb{R}$ is symmetric and vanishes on the diagonals, and $\epsilon_{i}$ 's are i.i.d. random variables with mean 0 and variance 1 . Note that $\mathbb{E} X(n)=0$.

In particular, Bai and Taqqu [1] studied limit theorems for normalized partial sum process of $X(n)$ :

$$
Y_{N}(t):=\frac{1}{A(N)} \sum_{n=1}^{[N t]} X(n)
$$

where [.] means integer part, and $A(N)$ is a suitable normalization factor. Depending on the behavior of $a(\cdot)$, the stationary process $X(n)$ may exhibit short or long memory.

As shown in Bai and Taqqu [1], in the short memory case, namely when the coefficient in (17) satisfies the summability condition

$$
\sum_{n=1}^{\infty} \sum_{0<i_{1}, \ldots, i_{k}<\infty}\left|a\left(i_{1}, \ldots, i_{k}\right) a\left(i_{1}+n, \ldots, i_{k}+n\right)\right|<\infty
$$

and $\mathbb{E}\left|\epsilon_{i}\right|^{2+\delta}<\infty$ for some $\delta>0$, the following central limit convergence as $N \rightarrow \infty$ holds:

$$
\frac{1}{N^{1 / 2}} \sum_{n=1}^{[N t]} X(n) \Rightarrow \sigma B(t)
$$

for some $\sigma \geq 0$, where $B(t)$ is a standard Brownian motion.

In the long memory case, assume that

$$
a(\cdot)=g(\cdot) L(\cdot) 1_{D^{c}}
$$

where

$$
D^{c}:=\left\{\left(i_{1}, \ldots, i_{k}\right): i_{p} \neq i_{q} \text { for } p \neq q\right\}
$$

guarantees that $a(\cdot)$ vanishes on the diagonals. The function $L(\cdot): \mathbb{Z}_{+}^{k} \rightarrow \mathbb{R}$ satisfie: 1

$$
\lim _{|\mathbf{i}| \rightarrow \infty} L(\mathbf{i})=1,
$$

and $g(\cdot): \mathbb{R}^{k} \rightarrow \mathbb{R}$ is the so-called generalized Hermite kernel of Class $(L)$.

Definition 3.1. A nonzero a.e. continuous function $g(\cdot): \mathbb{R}^{k} \rightarrow \mathbb{R}$ is called a generalized Hermite kernel of Class $(\mathrm{L})(\mathrm{GHK}(\mathrm{L}))$ if it satisfies

1. $g(\cdot)$ is homogeneous with exponent $\alpha$, namely, $g(\lambda \mathbf{x})=\lambda^{\alpha} g(\mathbf{x})$, for all $\lambda>0$, where

$$
\alpha \in\left(-\frac{k+1}{2},-\frac{k}{2}\right) ;
$$

\footnotetext{
${ }^{1}$ In Bai and Taqqu [1] eq. (25), $L(\cdot)$ is assumed to satisfy a slightly weaker condition than 22), that is, $\lim _{N \rightarrow \infty} L([N \mathbf{x}]+$ $\mathbf{B}(N))=1$ for any $\mathbf{x} \in \mathbb{R}_{+}^{k}$ and any bounded sequence $\mathbf{B}(N)$ in $\mathbb{Z}_{+}^{k}$ instead of $\lim _{\|\mathbf{x}\| \rightarrow \infty} L(\mathbf{x})=0$. Note that $L([N \mathbf{x}]+\mathbf{B}(N))$, $N \rightarrow \infty$, lets the argument increase in a specific band in the first quadrant, whereas $L(\mathbf{x}),\|\mathbf{x}\| \rightarrow \infty$, allows $\mathbf{x}$ to increase in an arbitrary way in the first quadrant. Here for simplicity we just assume [22), while the results stated here also hold under the weaker condition.
} 
2. The function $g(\cdot)$ satisfies the bound

$$
|g(\mathbf{x})| \leq g^{*}(\mathbf{x}):=c \sum_{j=1}^{m} x_{1}^{\gamma_{j 1}} \ldots x_{k}^{\gamma_{j k}},
$$

with the constant $c>0,-1<\gamma_{j l}<-1 / 2$ and $\sum_{l=1}^{k} \gamma_{j l}=\alpha$ for all $l=1, \ldots, m$.

If $g$ is a $\operatorname{GHK}(\mathrm{L})$, the following constant is well-defined (the integral is absolutely integrable)

$$
C_{g}=\int_{\mathbb{R}_{+}^{k}} g\left(x_{1}, \ldots, x_{k}\right) g\left(1+x_{1}, \ldots, 1+x_{k}\right) d x_{1} \ldots d x_{k},
$$

and $C_{g}>0$ always (Remark 3.6 of Bai and Taqqu [1]). Under this setup, Theorem 6.5 of Bai and Taqqu [1] showed that as $N \rightarrow \infty$,

$$
\frac{1}{N^{H}} \sum_{n=1}^{[N t]} X(n) \Rightarrow \int_{\mathbb{R}}^{\prime} \int_{0}^{t} g\left(s_{1}-x_{1}, \ldots, s_{k}-x_{k}\right) 1_{\left\{s_{1}>x_{1}, \ldots, s_{k}>x_{k}\right\}} W\left(d x_{1}\right) \ldots W\left(d x_{k}\right),
$$

where $W(\cdot)$ is the Brownian random measure, the prime ' indicates the exclusion of the diagonals $x_{p}=x_{q}$, $p \neq q$, and

$$
H=\alpha+\frac{k}{2}+1
$$

The limit in (26) was called a generalized Hermite process which generalizes the Hermite process (see, e.g., Dobrushin and Major [6] and Taqqu [13]) which corresponds to the special case $g(\mathbf{x})=x_{1}^{\alpha / k} \ldots x_{k}^{\alpha / k}$.

There is, however, a boundary case which the limit theorems (19) and (26) did not cover. This boundary case is as follows: set as in the long memory case

$$
a(\cdot)=g(\cdot) L(\cdot) 1_{D^{c}}
$$

where $D^{c}$ is as in (21),$L(\cdot)$ is as in (22), and $g$ is a function satisfying the assumptions in Definition 3.1 except that instead of assuming (23), the homogeneity exponent is set as $\alpha=-\frac{k+1}{2}$.

Remark 3.2. Note that if $\alpha<-\frac{k+1}{2}$, we are in the short memory regime. Indeed Proposition 5.4 of Bai and Taqqu [2] showed that $\alpha<-\frac{k+1}{2}$ implies (18), and thus (19) holds. So (28) is exactly the boundary case between short and long memory.

\subsection{Statement of the limit theorems}

Let throughout $\Rightarrow$ denote weak convergence in Skorohod space $D[0,1]$ with uniform metric. We shall show by the criterion formulated in Theorem [2.6, that a central limit theorem holds with an extra logarithmic factor in the normalization:

Theorem 3.3 (Nonlinear case). Let

$$
X(n)=\sum_{-\infty<i_{1}, \ldots, i_{k}<n} a\left(n-i_{1}, \ldots, n-i_{k}\right) \epsilon_{i_{1}} \ldots \epsilon_{i_{k}}
$$

as in (17) with $k \geq 2$ and the coefficient $a(\cdot)$ specified as in (27) where

$$
\alpha=-\frac{k+1}{2} \text {. }
$$

Assume also that $\mathbb{E}\left|\epsilon_{i}\right|^{3}<\infty$ and $C_{g}>0$. Then

$$
Y_{N}(t):=\frac{1}{\sqrt{N \ln N}} \sum_{n=1}^{[N t]} X(n) \Rightarrow \sigma B(t)
$$

where $\sigma=\sqrt{2 C_{g}}$, and $B(t)$ is a standard Brownian motion. 
Remark 3.4. Theorem 3.3 may be compared to a similar boundary case of limit theorems for nonlinear transform of long-memory Gaussian noise first considered in Breuer and Major [4] Theorem 1'. The proof there was done by a method of moments. See also Breton and Nourdin [3] who gave an alternative proof using the Malliavin calculus.

Note that to apply Theorem 2.6. the process $X(n)$ in (17) needs to have order $k \geq 2$. For completeness, we state also the corresponding result for linear process, namely, the case $k=1$ in Theorem 3.3, though the limit theorem for linear process is classical (see,e.g., Davydov [ 5$]$ ).

Theorem 3.5 (Linear case). Let

$$
X(n)=\sum_{-\infty<i<n} a(n-i) \epsilon_{i}
$$

where $a(n)=L(n) n^{-1}$ as $n \rightarrow \infty$, and let $L(n) \rightarrow c \neq 0$, and the i.i.d. standardized noise $\epsilon_{i}$ 's satisfy $\mathbb{E}\left|\epsilon_{i}\right|^{2+\delta}<\infty$ for some $\delta>0$. Then as $N \rightarrow \infty$,

$$
Y_{N}(t):=\frac{1}{\sqrt{N} \ln N} \sum_{n=1}^{[N t]} X(n) \Rightarrow \sigma B(t)
$$

where $\sigma=\sqrt{2}|c|$, and $B(t)$ is a standard Brownian motion.

\subsection{Proof of Theorem 3.3}

We first compute the asymptotic variance of the sum.

Lemma 3.6. Let $X(n)$ be given as in (17) with the coefficient specified as in (27) and $\alpha$ as in (28). Then $C_{g}$ defined in (25) is non-negative. If $C_{g}>0$, then as $N \rightarrow \infty$

$$
\mathbb{E}\left[\sum_{n=1}^{N} X(n)\right]^{2} \sim 2 C_{g} N \ln N
$$

If $C_{g}=0$, then

$$
\mathbb{E}\left[\sum_{n=1}^{N} X(n)\right]^{2}=o(N \ln N)
$$

Proof. Assume for simplicity $L(\cdot)=1$, and it is easy to extend the following arguments to the general case. First, since $g(\cdot)$ is homogeneous with exponent $\alpha=-k / 2-1 / 2$ by (28), one can write

$$
\begin{aligned}
\gamma(n): & =\mathbb{E} X(n) X(0)=\sum_{0<i_{1}, \ldots, i_{k}<\infty} g\left(i_{1}, \ldots, i_{k}\right) g\left(i_{1}+n, \ldots, i_{k}+n\right) 1_{D^{c}}\left(i_{1}, \ldots, i_{k}\right) \\
& =n^{-1} \sum_{0<i_{1}, \ldots, i_{k}<\infty} g\left(\frac{i_{1}}{n}, \ldots, \frac{i_{k}}{n}\right) g\left(\frac{i_{1}}{n}+1, \ldots, \frac{i_{k}}{n}+1\right) 1_{D^{c}}\left(i_{1}, \ldots, i_{k}\right) n^{-k} \\
& =n^{-1} \int_{\mathbb{R}_{+}^{k}} g\left(\frac{\left[n x_{1}\right]+1}{n}, \ldots, \frac{\left[n x_{k}\right]+1}{n}\right) g\left(\frac{\left[n x_{1}\right]+1}{n}+1, \ldots, \frac{\left[n x_{k}\right]+1}{n}+1\right) 1_{D^{c}}\left(\left[n x_{1}\right], \ldots,\left[n x_{k}\right]\right) d x_{1} \ldots d x_{k} \\
& =: n^{-1} C_{n}(g) .
\end{aligned}
$$

Because the bounding function $g^{*}$ in Definition 3.1 is decreasing in every variable, the absolute of the integrand above is bounded by

$$
g^{*}\left(x_{1}, \ldots, x_{k}\right) g^{*}\left(x_{1}+1, \ldots, x_{k}+1\right)=c^{2} \sum_{j_{1}, j_{2}=1}^{m} x_{1}^{\gamma_{j_{1}, 1}}\left(x_{1}+1\right)^{\gamma_{j_{2}, 1}} \ldots x_{k}^{\gamma_{j_{1}, k}}\left(x_{k}+1\right)^{\gamma_{j_{2}, k}}
$$


which is integrable on $\mathbb{R}_{+}^{k}$ because all $\gamma_{p, q} \in(-1,-1 / 2)$ and

$$
\int_{\mathbb{R}_{+}} x^{\gamma}(x+1)^{\gamma^{\prime}} d x<\infty \quad \text { for any }-1<\gamma, \gamma^{\prime}<-1 / 2
$$

Since $g$ is assumed to be a.e. continuous, by the Dominated Convergence Theorem, as $n \rightarrow \infty$ we have

$$
C_{n}(g) \rightarrow C_{g}:=\int_{\mathbb{R}^{k}} g\left(x_{1}, \ldots, x_{k}\right) g\left(x_{1}+1, \ldots, x_{k}+1\right) d x_{1} \ldots d x_{k} .
$$

Hence when $C_{n} \neq 0$, one has when $n>0$

$$
\gamma(n) \sim n^{-1} C_{g},
$$

and when $C_{n}=0$, one has

$$
\gamma(n)=o\left(n^{-1}\right) .
$$

We shall use the fact that if $a_{n} \sim n^{-1}$ as $n \rightarrow \infty$, then $\sum_{n=1}^{N} a_{n} \sim \ln N$ as $N \rightarrow \infty$. So when $C_{g} \neq 0$, one has

$$
\mathbb{E}\left[\sum_{n=1}^{N} X(n)\right]=\sum_{n_{1}, n_{2}=1}^{N} \gamma\left(n_{1}-n_{2}\right)=N \sum_{n=-N+1}^{N-1} \gamma(n)-\sum_{n=-N+1}^{N-1}|n| \gamma(n) \sim 2 C_{g} N \ln N
$$

Note that since $\gamma(n) \sim n^{-1} C_{g}$, the term $\sum_{n=-N+1}^{N-1}|n| \gamma(n) \sim 2 C_{g} N$ and is thus negligible.

The preceding asymptotic equivalence also shows that if $C_{g} \neq 0$ then $C_{g}>0$ because the variance is non-negative.

If $C_{g}=0$, following similar lines of argument, one gets (29).

Lemma 3.7. Define the mapping $(\cdot, \cdot)_{0}: \mathbb{R}^{2} \rightarrow \mathbb{R}$ as

$$
\left(x_{1}, x_{2}\right)_{0}= \begin{cases}\left|x_{1}-x_{2}\right| & \text { if } x_{1} \neq x_{2} \\ 1 & \text { if } x_{1}=x_{2}=x .\end{cases}
$$

For $-1<\gamma_{1}, \gamma_{2}<-1 / 2$ and $n_{1}, n_{2} \in\{1,2, \ldots\}$, we have for some constant $C>0$ not depending on $n_{1}, n_{2}$ that

$$
\sum_{p \in \mathbb{Z}}\left(n_{1}-p\right)_{+}^{\gamma_{1}}\left(n_{2}-p\right)_{+}^{\gamma_{2}} \leq C\left(n_{1}, n_{2}\right)_{0}^{\gamma_{1}+\gamma_{2}+1} .
$$

Proof. For the case $n_{1}=n_{2}=n$, choose $C=\sum_{p<n}(n-p)^{\gamma_{1}+\gamma_{2}}<\infty$ since $\gamma_{1}+\gamma_{2}<-1$. When $n_{1} \neq n_{2}$, suppose that $n_{1}<n_{2}$. Then

$$
\begin{aligned}
\sum_{p \in \mathbb{Z}}\left(n_{1}-p\right)_{+}^{\gamma_{1}}\left(n_{2}-p\right)_{+}^{\gamma_{2}} & =\sum_{p=1}^{\infty} p^{\gamma_{1}}\left(n_{2}-n_{1}+p\right)_{2}^{\gamma} \leq \int_{0}^{\infty} x^{\gamma_{1}}\left(n_{2}-n_{1}+x\right)^{\gamma_{2}} d x \\
& =\left(n_{2}-n_{1}\right)^{\gamma_{1}+\gamma_{2}+1} \int_{0}^{\infty} y^{\gamma_{1}}(1+y)^{\gamma_{2}} d y
\end{aligned}
$$

where the integral converges.

The following simple fact will be used.

Lemma 3.8. Suppose that $\gamma_{j}<-1 / 2$ for all $j=1, \ldots, k, k \geq 2$, and $\gamma_{1}+\ldots+\gamma_{k} \geq-k / 2-1 / 2$. Then

$$
-\frac{r}{2}-\frac{1}{2}<\gamma_{1}+\ldots+\gamma_{r}<-\frac{r}{2} \quad \text { for all } r=1, \ldots, k-1 .
$$

In addition, each $\gamma_{j}>-1, j=1, \ldots, k$. 
Proof. The inequality $\gamma_{1}+\ldots+\gamma_{r}<-\frac{r}{2}$ is obvious. For the other inequality, suppose that $\gamma_{1}+\ldots+\gamma_{r} \leq$ $-r / 2-1 / 2$ for some $r \in\{1, \ldots, k\}$. Because $\gamma_{r+1}, \ldots, \gamma_{k}<-1 / 2$, we get the contradiction: $\gamma_{1}+\ldots+\gamma_{k}<$ $-r / 2-1 / 2-(k-r) / 2=-k / 2-1 / 2$.

Then we show by contradiction that each $\gamma_{j}>-1$. Suppose, e.g., $\gamma_{k} \leq-1$. By what was just proved, one has $\gamma_{1}+\ldots+\gamma_{k-1}<-(k-1) / 2$. Thus by adding $\gamma_{k} \leq-1$, one gets $\gamma_{1}+\ldots+\gamma_{k}<-k / 2-1 / 2$, which contradicts the assumption.

We need the following lemma, which is a consequence of Corollary 1.1 (b) of Terrin and Taqqu [14].

Lemma 3.9. If $\alpha_{1}, \ldots, \alpha_{m}, m \geq 2$, satisfy

$$
\alpha_{1}, \ldots, \alpha_{n}>-1, \quad \sum_{i=1}^{m} \alpha_{i}+m>1,
$$

then for any $c>0$

$$
\int_{[0, c]^{m}}\left|x_{1}-x_{2}\right|^{\alpha_{1}}\left|x_{2}-x_{3}\right|^{\alpha_{2}} \ldots\left|x_{m-1}-x_{m}\right|^{\alpha_{m-1}}\left|x_{m}-x_{1}\right|^{\alpha_{m}} d x_{1} \ldots d x_{m}<\infty .
$$

We need also the following hypercontractivity inequality for proving tightness in $D[0,1]$ (Proposition 5.2 of Bai and Taqqu [1])

Lemma 3.10. Suppose that $h \in \ell^{2}\left(\mathbb{Z}^{k}\right)$ vanishing on the diagonals. Let $X=\sum_{\mathbf{i} \in \mathbb{Z}^{k}} h(\mathbf{i}) \prod_{p=1}^{k} \epsilon_{i_{p}}, k \geq 1$. If for some $p^{\prime}>p>2, \mathbb{E}\left|\epsilon_{i}\right|^{p^{\prime}}<\infty$, then one has for some constant $c_{p, k}>0$ which does not depend on $h$ that

$$
\mathbb{E}\left[|X|^{p}\right]^{1 / p} \leq c_{p, k} \mathbb{E}\left[|X|^{2}\right]^{1 / 2}
$$

Proof of Theorem 3.3. Let $C>0$ be a constant whose value can change from line to line. We first show that the finite-dimensional distributions of $Y_{N}(t)$ converges to those of $\sigma B(t)$ using Theorem 2.6. First, the convergence of the covariance structure of $Y_{N}(t)$ to that of $\sigma B(t)$ follows from Lemma 3.6 the fact that for $s \leq t$ we have

$$
\mathbb{E} Y_{N}(t) Y_{N}(s)=\frac{1}{2}\left[\mathbb{E} Y_{N}(t)^{2}+\mathbb{E} Y_{N}(s)^{2}-\mathbb{E}\left(Y_{N}(t)-Y_{N}(s)\right)^{2}\right] \sim \frac{1}{2}\left[\mathbb{E} Y_{N}(t)^{2}+\mathbb{E} Y_{N}(s)^{2}-\mathbb{E} Y_{N}(t-s)^{2}\right]
$$

as $N \rightarrow \infty$, since $X(n)$ is stationary. We now check the contraction conditions (14). For simplicity we set $L(\cdot)=1$ and $t=1$. We can write

$$
Y_{N}(1)=\sum_{-\infty<i_{1}, \ldots, i_{k}<+\infty} f_{N}\left(i_{1}, \ldots, i_{k}\right) \epsilon_{i_{1}} \ldots \epsilon_{i_{k}}
$$

where

$$
f_{N}\left(i_{1}, \ldots, i_{k}\right)=\frac{1}{\sqrt{N \ln N}} \sum_{n=1}^{N} g\left(n-i_{1}, \ldots, n-i_{k}\right) 1_{D^{c} \cap\left\{i_{1}<n, \ldots, i_{k}<n\right\}} .
$$

To simplify notation, we set

$$
\begin{gathered}
\mathbf{p}=\left(p_{1}, \ldots, p_{r}\right), \quad \mathbf{q}=\left(q_{1}, \ldots, q_{k-r}\right), \\
\mathbf{i}_{1}=\left(i_{1}, \ldots, i_{k-r}\right), \quad \mathbf{i}_{2}=\left(i_{k-r+1}, \ldots, i_{2 k-2 r}\right), \quad \mathbf{i}=\left(\mathbf{i}_{1}, \mathbf{i}_{2}\right),
\end{gathered}
$$

and let $\mathbf{1}$ stand for a vector of 1's of suitable dimension. We also use the convention that $\mathbf{x}^{\mathbf{a}}=x_{1}^{a_{1}} \ldots x_{n}^{a_{n}}$ if $\mathbf{x}=\left(x_{1}, \ldots, x_{n}\right)$ and $\mathbf{a}=\left(a_{1}, \ldots, a_{n}\right)$. Let $(\Sigma \mathbf{x})=x_{1}+\ldots+x_{n}$ if $\mathbf{x}=\left(x_{1}, \ldots, x_{n}\right)$.

Set $g^{*}(\cdot)$ be as in Definition 3.1 which we write by splitting $\mathbf{x}=\left(\mathbf{x}_{1}, \mathbf{x}_{2}\right)$, where $\mathbf{x}_{1} \in \mathbb{R}_{+}^{r}$ and $\mathbf{x}_{2} \in \mathbb{R}_{+}^{k-r}$ :

$$
g^{*}\left(\mathbf{x}_{1}, \mathbf{x}_{2}\right)=c \sum_{j=1}^{m} \mathbf{x}_{1}^{\boldsymbol{\beta}_{j}} \mathbf{x}_{2}^{\boldsymbol{\eta}_{j}}, \quad \boldsymbol{\beta}_{j}=\left(\gamma_{j 1}, \ldots, \gamma_{j r}\right), \quad \boldsymbol{\eta}_{j}=\left(\gamma_{j, r+1}, \ldots, \gamma_{j k}\right),
$$


so that

$$
\sum_{i=1}^{r} \beta_{j i}+\sum_{i=1}^{k-r} \eta_{j i}=\sum_{i=1}^{k} \gamma_{j i}=\alpha
$$

which we write simply as $\sum \boldsymbol{\beta}+\sum \boldsymbol{\eta}=\sum \boldsymbol{\gamma}=\alpha$. For convenience, if some component $x_{j}$ of $\mathbf{x}$ is negative, we set $\mathbf{x}^{a}=0$ and hence $g^{*}(\mathbf{x})=0$. Then in view of (31), (11) and (24),

$$
\begin{aligned}
& \left|\left(f_{N} \star_{r} f_{N}\right)(\mathbf{i})\right| \leq \frac{1}{N \ln N} \sum_{n_{1}, n_{2}=1}^{N} \sum_{\mathbf{p}} g^{*}\left(n_{1} \mathbf{1}-\mathbf{p}, n_{1} \mathbf{1}-\mathbf{i}_{1}\right) g^{*}\left(n_{2} \mathbf{1}-\mathbf{p}, n_{2} \mathbf{1}-\mathbf{i}_{2}\right) \\
& =\frac{c^{2}}{N \ln N} \sum_{n_{1}, n_{2}=1}^{N} \sum_{j_{1}, j_{2}=1}^{m}\left(n_{1} \mathbf{1}-\mathbf{i}_{1}\right)^{\boldsymbol{\eta}_{j_{1}}}\left(n_{2} \mathbf{1}-\mathbf{i}_{2}\right)^{\boldsymbol{\eta}_{j_{2}}} \sum_{\mathbf{p}}\left(n_{1} \mathbf{1}-\mathbf{p}\right)^{\boldsymbol{\beta}_{j_{1}}}\left(n_{2} \mathbf{1}-\mathbf{p}\right)^{\boldsymbol{\beta}_{j_{2}}},
\end{aligned}
$$

by using (32). By Lemma 3.7, we have for the last sum,

$$
\sum_{\mathbf{p}}\left(n_{1} \mathbf{1}-\mathbf{p}\right)^{\boldsymbol{\beta}_{j_{1}}}\left(n_{2} \mathbf{1}-\mathbf{p}\right)^{\boldsymbol{\beta}_{j_{2}}}=\sum_{p_{1}, \ldots, p_{r}} \prod_{u=1}^{r}\left(n_{1}-p_{u}\right)^{\gamma_{j_{1}, u}} \prod_{v=1}^{r}\left(n_{1}-p_{v}\right)^{\gamma_{j_{2}, v}} \leq C\left(n_{1}, n_{2}\right)_{0}^{\left(\Sigma \boldsymbol{\beta}_{j_{1}}\right)+\left(\Sigma \boldsymbol{\beta}_{j_{2}}\right)+r} .
$$

Hence

$$
\begin{aligned}
& \left\|f_{N} \star_{r} f_{N}\right\|_{2 k-2 r}^{2}=\sum_{\mathbf{i}}\left[\left(f_{N} \star_{r} f_{N}\right)(\mathbf{i})\right]^{2} \\
& \leq \frac{C}{N^{2}(\ln N)^{2}} \sum_{\mathbf{i}}\left(\sum_{n_{1}, n_{2}=1}^{N} \sum_{j_{1}, j_{2}=1}^{m}\left(n_{1}, n_{2}\right)_{0}^{\left(\Sigma \boldsymbol{\beta}_{j_{1}}\right)+\left(\Sigma \boldsymbol{\beta}_{j_{2}}\right)+r}\left(n_{1} \mathbf{1}-\mathbf{i}_{1}\right)^{\boldsymbol{\eta}_{j_{1}}}\left(n_{2} \mathbf{1}-\mathbf{i}_{2}\right)^{\boldsymbol{\eta}_{j_{2}}}\right)^{2} \\
& =\frac{C}{N^{2}(\ln N)^{2}} \sum_{j_{1}, j_{2}, j_{3}, j_{4}=1}^{m} \sum_{n_{1}, n_{2}, n_{3}, n_{4}=1}^{N}\left(n_{1}, n_{2}\right)_{0}^{\left(\Sigma \boldsymbol{\beta}_{j_{1}}\right)+\left(\Sigma \boldsymbol{\beta}_{j_{2}}\right)+r}\left(n_{3}, n_{4}\right)_{0}^{\left(\Sigma \boldsymbol{\beta}_{j_{3}}\right)+\left(\Sigma \boldsymbol{\beta}_{j_{4}}\right)+r} \\
& \times \sum_{\mathbf{i}_{1}}\left(n_{1} \mathbf{1}-\mathbf{i}_{1}\right)^{\boldsymbol{\eta}_{j_{1}}}\left(n_{3} \mathbf{1}-\mathbf{i}_{1}\right)^{\boldsymbol{\eta}_{j_{3}}} \sum_{\mathbf{i}_{2}}\left(n_{2} \mathbf{1}-\mathbf{i}_{2}\right)^{\boldsymbol{\eta}_{j_{2}}}\left(n_{4} \mathbf{1}-\mathbf{i}_{2}\right)^{\boldsymbol{\eta}_{j_{4}}} \\
& \leq \frac{C}{N^{2}(\ln N)^{2}} \sum_{j_{1}, j_{2}, j_{3}, j_{4}=1}^{m} \sum_{n_{1}, n_{2}, n_{3}, n_{4}=1}^{N}\left(n_{1}, n_{2}\right)_{0}^{\left(\Sigma \boldsymbol{\beta}_{j_{1}}\right)+\left(\Sigma \boldsymbol{\beta}_{j_{2}}\right)+r}\left(n_{3}, n_{4}\right)_{0}^{\left(\Sigma \boldsymbol{\beta}_{j_{3}}\right)+\left(\Sigma \boldsymbol{\beta}_{j_{4}}\right)+r} \\
& \times\left(n_{1}, n_{3}\right)_{0}^{\left(\Sigma \boldsymbol{\eta}_{j_{1}}\right)+\left(\Sigma \boldsymbol{\eta}_{j_{3}}\right)+k-r}\left(n_{2}, n_{4}\right)_{0}^{\left(\Sigma \boldsymbol{\eta}_{j_{2}}\right)+\left(\Sigma \boldsymbol{\eta}_{j_{4}}\right)+k-r}
\end{aligned}
$$

where we have applied again Lemma 3.7 to get the last inequality. Note that if one adds up the power exponents in the last expression, one gets

$$
\left(\Sigma \boldsymbol{\beta}_{j_{1}}\right)+\left(\Sigma \boldsymbol{\eta}_{j_{1}}\right)+\left(\Sigma \boldsymbol{\beta}_{j_{2}}\right)+\left(\Sigma \boldsymbol{\eta}_{j_{2}}\right)+\left(\Sigma \boldsymbol{\beta}_{j_{3}}\right)+\left(\Sigma \boldsymbol{\eta}_{j_{3}}\right)+\left(\Sigma \boldsymbol{\beta}_{j_{4}}\right)+\left(\Sigma \boldsymbol{\eta}_{j_{4}}\right)+2 k=4 \alpha+2 k=-2,
$$

by (33), where the last equality of (35) is due to assumption (28).

Note also that by Lemma 3.8, we have for $r \in\{1, \ldots, k-1\}$ that

$$
-\frac{r}{2}-\frac{1}{2}<\left(\Sigma \boldsymbol{\beta}_{j_{1}}\right),\left(\Sigma \boldsymbol{\beta}_{j_{2}}\right),\left(\Sigma \boldsymbol{\beta}_{j_{3}}\right),\left(\Sigma \boldsymbol{\beta}_{j_{4}}\right)<-\frac{r}{2}
$$

and

$$
-\frac{k-r}{2}-\frac{1}{2}<\left(\Sigma \boldsymbol{\eta}_{j_{1}}\right),\left(\Sigma \boldsymbol{\eta}_{j_{3}}\right),\left(\Sigma \boldsymbol{\eta}_{j_{2}}\right),\left(\Sigma \boldsymbol{\eta}_{j_{4}}\right)<-\frac{k-r}{2} .
$$

Let $\alpha_{1}=\left(\sum \beta_{j_{1}}\right)+\left(\sum \beta_{j_{2}}\right)+r$ be the exponent of $\left(n_{1}, n_{2}\right)_{0}$ in (34). Then

$$
-1=-r / 2-1 / 2-r / 2-1 / 2+r<\alpha_{1}<-r / 2-r / 2+r=-r+r=0 .
$$

Define similarly $\alpha_{2}, \alpha_{3}, \alpha_{4}$ for the other exponents in (34), which all lie strictly between -1 and 0 . Hence, the convergence

$$
\lim _{N \rightarrow \infty}\left\|f_{N} \star_{r} f_{N}\right\|_{2 k-2 r}^{2}=0, \quad r=1, \ldots, k-1,
$$


will follow if one shows that

$$
\sup _{N} N^{-2} \sum_{n_{1}, n_{2}, n_{3}, n_{4}=1}^{N}\left(n_{1}, n_{2}\right)_{0}^{\alpha_{1}}\left(n_{2}, n_{3}\right)_{0}^{\alpha_{2}}\left(n_{3}, n_{4}\right)_{0}^{\alpha_{3}}\left(n_{4}, n_{1}\right)_{0}^{\alpha_{4}}<\infty
$$

where by (35)

$$
-1<\alpha_{j}<0, j=1, \ldots 4, \quad \alpha_{1}+\alpha_{2}+\alpha_{3}+\alpha_{4}=-2 .
$$

Let's consider first the sum in (37) over only distinct $n_{1}, \ldots, n_{4}$ (we use the prime' to indicate that the sum does not include the diagonals). In this case,

$$
\begin{gathered}
\sum_{1 \leq n_{1}, n_{2}, n_{3}, n_{4} \leq N}^{\prime}\left|\frac{n_{1}}{N}-\frac{n_{2}}{N}\right|^{\alpha_{1}}\left|\frac{n_{2}}{N}-\frac{n_{3}}{N}\right|^{\alpha_{2}}\left|\frac{n_{3}}{N}-\frac{n_{4}}{N}\right|^{\alpha_{3}}\left|\frac{n_{4}}{N}-\frac{n_{1}}{N}\right|^{\alpha_{4}} N^{-4}=\int\left|\frac{\left[N x_{1}\right]-\left[N x_{2}\right]}{N}\right|^{\alpha_{1}} \times \\
\left|\frac{\left[N x_{2}\right]-\left[N x_{3}\right]}{N}\right|^{\alpha_{2}}\left|\frac{\left[N x_{3}\right]-\left[N x_{4}\right]}{N}\right|^{\alpha_{3}}\left|\frac{\left[N x_{4}\right]-\left[N x_{1}\right]}{N}\right|^{\alpha_{4}} I\left\{N^{-1} \leq x_{i} \leq 1+N^{-1},\left[N x_{i}\right] \neq\left[N x_{j}\right], \forall i \neq j\right\} d \mathbf{x} .
\end{gathered}
$$

Note that for any $x, y>0$, one has that $|[N x]-[N y]|=n$ implies that $|N x-N y| \leq n+1$ which implies $|x-y| \leq(n+1) / N$, for $n \geq 0$. Then since each $\alpha<0$, we get

$$
\sup _{N}\left|\frac{[N x]-[N y]}{N}\right|^{\alpha}|x-y|^{-\alpha} I\{[N x] \neq[N y]\} \leq \sup _{|[N x]-[N y]|=n, n \in \mathbb{Z}_{+}}\left(\frac{n}{N}\right)^{\alpha}\left(\frac{n+1}{N}\right)^{-\alpha}=\sup _{n \in \mathbb{Z}_{+}}\left(\frac{n+1}{n}\right)^{-\alpha}=2^{-\alpha} .
$$

Hence the the sum in (37) over distinct $n_{1}, \ldots, n_{4}$ is bounded by

$$
C \int_{[0,2]^{4}}\left|x_{1}-x_{2}\right|^{\alpha_{1}}\left|x_{2}-x_{3}\right|^{\alpha_{2}}\left|x_{3}-x_{4}\right|^{\alpha_{3}}\left|x_{4}-x_{1}\right|^{\alpha_{4}} d x_{1} d x_{2} d x_{3} d x_{4},
$$

which is finite due to Lemma 3.9

Consider now the the sum in (37) over $n_{1}, \ldots, n_{4}$ with only three of them distinct. Let, for example, $n_{1}=n_{4}$, and we need to show that the following

$$
\begin{aligned}
& \sup _{N} N^{-2} \sum_{1 \leq n_{1}, n_{2}, n_{3} \leq N}^{\prime}\left|n_{1}-n_{2}\right|^{\alpha_{1}}\left|n_{2}-n_{3}\right|^{\alpha_{2}}\left|n_{3}-n_{1}\right|^{\alpha_{3}}= \\
& \sup _{N} N^{1+\alpha_{1}+\alpha_{2}+\alpha_{3}} \sum_{1 \leq n_{1}, n_{2}, n_{3} \leq N}^{\prime}\left|\frac{n_{1}}{N}-\frac{n_{2}}{N}\right|^{\alpha_{1}}\left|\frac{n_{2}}{N}-\frac{n_{3}}{N}\right|^{\alpha_{2}}\left|\frac{n_{3}}{N}-\frac{n_{1}}{N}\right|^{\alpha_{3}} N^{-3}<\infty .
\end{aligned}
$$

Note that (38) entails that $-2<\alpha_{1}+\alpha_{2}+\alpha_{3}<-1$. Then $N^{1+\alpha_{1}+\alpha_{2}+\alpha_{3}} \rightarrow 0$ as $N \rightarrow \infty$, and the boundedness of the multiple sum can be established similarly as above using integral approximation and Lemma 3.9 .

If the sum in (37) is over $n_{1}, \ldots, n_{4}$ with only two or less of them distinct, the boundedness is easily established through bounding all the summands by one constant, because we have the factor $N^{-2}$.

So (37) holds and thus (36) holds, and the convergence of finite-dimensional distributions is proved.

Now we show tightness. By Lemma 3.10, one can choose $p \in(2,3)$, so that by Lemma 3.6 if $0<s<t<1$, one has for $N$ large enough,

$$
\begin{aligned}
\mathbb{E}\left|Y_{N}(t)-Y_{N}(s)\right|^{p} & \leq C\left[\mathbb{E}\left|Y_{N}(t)-Y_{N}(s)\right|^{2}\right]^{p / 2} \leq C\left[\frac{[N t]-[N s]}{N} \cdot \frac{\ln ([N t]-[N s])}{\ln N}\right]^{p / 2} \\
& \leq C\left[\frac{[N t]-[N s]}{N}\right]^{p / 2-\delta}
\end{aligned}
$$

where $\delta>0$ is small enough so that $p / 2-\delta>1$. The last inequality is true because $\ln x$ is slowly varying as $x \rightarrow \infty$ and so one applies the Potter's bound (see e.g., equation (2.3.6) of Giraitis et al. [7]). Note that $F_{N}(t):=[N t] / N$ is a non-decreasing right continuous function on $[0,1]$ and that $F_{N}$ converges uniformly to $F(t):=t$ as $N \rightarrow \infty$. Hence by Lemma 4.4.1 and Theorem 4.4.1 of Giraitis et al. []], the tightness in $D[0,1]$ is proved. 


\subsection{Proof of Theorem 3.5}

Proof. Set for simplicity $L(n)=c$. The covariance $\gamma(n)$ for $n>0$ is

$$
\gamma(n)=\mathbb{E} X(n) X(0)=\sum_{i=1}^{\infty} a_{i+n} a_{i}=c^{2} \sum_{i=1}^{\infty}(i+n)^{-1} i^{-1}
$$

Note that as $n \rightarrow \infty$,

$$
\sum_{i=2}^{\infty}(i+n)^{-1} i^{-1}=n^{-1} \sum_{i=2}^{\infty}\left(\frac{i}{n}+1\right)^{-1}\left(\frac{i}{n}\right)^{-1} \frac{1}{n}=n^{-1} \int_{2 / n}^{\infty}\left(\frac{[n x]}{n}+1\right)^{-1}\left(\frac{[n x]}{n}\right)^{-1} d x \sim n^{-1} \ln n .
$$

The last asymptotic can be seen from:

$$
\int_{2 / n}^{\infty}(x+1)^{-1} x^{-1} d x \leq \int_{2 / n}^{\infty}\left(\frac{[n x]}{n}+1\right)^{-1}\left(\frac{[n x]}{n}\right)^{-1} d x \leq \int_{1 / n}^{\infty}(y+1)^{-1} y^{-1} d y
$$

where we have used the fact $x-1 / n \leq[n x] / n \leq x$, and both the lower and upper bounds are asymptotically equivalent to $\ln n$ as $n \rightarrow \infty$.

Hence

$$
\gamma(n) \sim c^{2} n^{-1} \ln n \quad \text { as } n \rightarrow \infty
$$

So as $N \rightarrow \infty$, one has

$$
\begin{aligned}
\mathbb{E}\left(\sum_{n=1}^{N} X(n)\right)^{2} & =N \sum_{n=-N+1}^{N-1} \gamma(n)-\sum_{n=-N+1}^{N-1}|n| \gamma(n) \\
& \sim 2 c^{2} N \sum_{n=1}^{N} n^{-1} \ln n \sim 2 c^{2} N \int_{1}^{N} x^{-1} \ln x d x \sim 2 c^{2} N(\ln N)^{2}
\end{aligned}
$$

Note that by (39) the term $\sum_{n=-N+1}^{N-1}|n| \gamma(n)=O(N \ln N)$ and is thus negligible. Having obtained the asymptotic variance (40), the proof is then concluded by applying Davydov [5] Theorem 2 (though this theorem was stated for a linearly interpolated version of $Y_{N}(t)$ in the space $C[0,1]$, it is straightforward to adapt the the proof, which consists of showing convergence of finite-dimensional distributions and establishing tightness by moment estimate, to establish convergence in $D[0,1]$ with the uniform metric.)

Remark 3.11. One may wonder if it is possible to get a different normalization in the nonlinear case in Theorem 3.3, since the normalization in the linear case in Theorem 3.5 has an extra $\sqrt{\ln N}$ factor. This is not possible under our setting where the kernel $g$ is homogeneous with exponent $\alpha$ and is bounded by a linear combination of products of purely power functions $x_{1}^{\gamma_{1}} \ldots x_{k}^{\gamma_{k}}$, where each $\gamma_{j}<-1 / 2$ and $\gamma_{1}+\ldots+\gamma_{k}=\alpha$.

Indeed, if one wanted to get some extra logarithmic factor in the covariance $\gamma(n)$, one would set for example $g\left(x_{1}, \ldots, x_{k}\right)=x_{1}^{\gamma_{1}} \ldots x_{k}^{\gamma_{k}}$ with $\gamma_{k}=-1$. But this will not achieve the stated goal. Indeed, by Lemma 3.8, using contradiction, we have $\alpha=\gamma_{1}+\ldots+\gamma_{k}<-k / 2-1 / 2$, which falls into the short memory regime (see Remark 3.2) and thus the normalization is $\sqrt{N}$ as in (19).

Acknowledgments. This work was partially supported by the NSF grant DMS-1309009 at Boston University. We would also like to thank the referee for his comments.

\section{References}

[1] S. Bai and M.S. Taqqu. Generalized Hermite processes, discrete chaos and limit theorems. Stochastic Processes and Their Applications, 124(4):1710-1739, 2014.

[2] S. Bai and M.S. Taqqu. Convergence of long-memory discrete k-th order Volterra processes. Stochastic Processes and Their Applications, 125(5):2026-2053, 2015. 
[3] J.C. Breton and I. Nourdin. Error bounds on the non-normal approximation of hermite power variations of fractional brownian motion. Electronic Communications in Probability, 13:482-493, 2008.

[4] P. Breuer and P. Major. Central limit theorems for non-linear functionals of Gaussian fields. Journal of Multivariate Analysis, 13(3):425-441, 1983.

[5] Y.A. Davydov. The invariance principle for stationary processes. Theory of Probability and Its Applications, 15(3):487-498, 1970.

[6] R.L. Dobrushin and P. Major. Non-central limit theorems for non-linear functional of Gaussian fields. Probability Theory and Related Fields, 50(1):27-52, 1979.

[7] L. Giraitis, H.L. Koul, and D. Surgailis. Large Sample Inference for Long Memory Processes. World Scientific Publishing Company Incorporated, 2012.

[8] I. Nourdin and G. Peccati. Normal Approximations With Malliavin Calculus: From Stein's Method to Universality. Cambridge Tracts in Mathematics. Cambridge University Press, 2012.

[9] I Nourdin, G. Peccati, and G Reinert. Invariance principles for homogeneous sums: universality of Gaussian Wiener chaos. The Annals of Probability, 38(5):1947-1985, 2010.

[10] G. Peccati and M.S. Taqqu. Wiener Chaos: Moments, Cumulants and Diagrams: a Survey With Computer Implementation. Springer Verlag, 2011.

[11] G. Peccati and C. Zheng. Universal Gaussian fluctuations on the discrete Poisson chaos. Bernoulli, 20 (2):697-715, 2014.

[12] V.I. Rotar. Limit theorems for polylinear forms. Journal of Multivariate analysis, 9(4):511-530, 1979.

[13] M.S. Taqqu. Convergence of integrated processes of arbitrary Hermite rank. Probability Theory and Related Fields, 50(1):53-83, 1979.

[14] N. Terrin and M.S. Taqqu. Power counting theorem in Euclidean space. In Random Walks, Brownian Motion, and Interacting Particle Systems, pages 425-440. Springer Verlag, 1991. 